\title{
Algorithms for the Reduction of Surface Evolution Discretization Error
}

\author{
Hernan A. Rueda and Mark E. Law \\ Department of Electrical Engineering, University of Florida \\ Gainesville, FL 32611 \\ Phone: 904-392-6276 Fax: 904-392-8381 \\ har@tcad.ee.ufl.edu law@tcad.ee.ufl.edu
}

\begin{abstract}
This paper investigates numerical error due to time and spatial discretization. The relative error contributions of each discretization scheme is examined. Two algorithms are then introduced to control the error due to each modelling discretization scheme.
\end{abstract}

\section{Introduction}

Surface evolution algorithms[1-4] all suffer from some numerical error as a function of both the time and spatial discretization. We have studied both components of the error, and conclude that the spatial discretization is the largest component. We suggest techniques that can control the error from both time and spatial discretization.

\section{Time Discretization}

The velocity of etch or deposition of any node on a surface may be dependent on the node's current position on the surface and the current time in the process, $V(x, t)$. In general terms, this may be summarized by the following equations:

$$
\begin{gathered}
\vec{V} \propto x, t \\
x_{1}-x_{0}=\int_{t_{0}}^{t_{1}} \vec{V}(x, t) d t
\end{gathered}
$$

When solving this integration, one may use different approaches computationally. Traditionally, in most surface evolution simulators[1-4], a "forward euler approach (FE)" has been implemented:

$$
\begin{gathered}
x_{1}=x_{0}+\Delta t \cdot \vec{V}\left(x_{0}, t_{0}\right) \\
\Delta t=t_{1}-t_{0}
\end{gathered}
$$


The new position of the discretized surface node is computed by adding the old position to the product of the time step and the velocity vector. This algorithm has the advantage of simplicity since all the parameters may be determined at any given initial position for a surface node. However, the error introduced by the equation depends on the rate of change of the velocity. For an isotropic etch or deposition it is accurate, but inaccurate in an anisotropic simulation, where the velocity vector is changing in magnitude and/or direction. For this algorithm, the error may be controlled by choosing a very small time step continuously throughout the simulation, in hopes that the change in velocity vector for the given node changes negligibly during that time interval. However, this wastes CPU time, since at times throughout the simulation, the velocity vectors of all the surface nodes may not change. Also, the correct size of the time step to take is difficult to compute.

Two algorithms have been investigated to reduce this quantization error. The first is the "trapezoidal rule (TR)" algorithm:

$$
x_{1}=x_{0}+\Delta t \cdot\left[\frac{\vec{V}\left(x_{0}, t_{0}\right)+\vec{V}\left(x_{1}, t_{1}\right)}{2}\right]
$$

This procedure calculates the new node position by computing the average of the initial and final velocities in the given time interval. It then also uses the initial and final velocity vectors to calculate an appropriate succeeding time step. This adaptive time step algorithm can then control the time discretization error. The disadvantage of using this algorithm is that it is more computationally intensive because the new velocity must be solved iteratively.

The differences between these methods greatly depends on the grid resolution of the test structures. For fine grids the differences between these methods is much smaller than coarse grid surfaces. Figure 1 summarizes these results for different surface evolutions. In general, the error from the time discretization is less than the error from the spatial discretization.

\section{Spatial Discretization}

This led to the development of a surface grid refining technique (SGR) for etching and deposition modeling. This technique uses the radius of curvature at each given node on the surface to determine whether new nodes should be placed next to that node to help define the surface local to the node. When the radius is less than a specified length, new neighboring nodes are then added. This technique allows more defined corners to be evolved. It proves to be very helpful in both isotropic and anisotropic process modelling.

When the grid refining technique is coupled with the adaptive time step procedure, an initial coarse grid will not affect the surface development of the simulation. An initially coarse grid simulation will then eventually result in the similar final surface structure and therefore less discretization error as compared to a very fine grid.

Figure 2 shows how the result of an anisotropic deposition process, using both error reduction algorithms, with an initial grid spacing of 0.10 um can resemble that of a 0.01 um grid.

Figure 3 shows the relative differences among the different surface evolution algorithms at 0.10 um spacing and how they compare to the 0.01 um grid simulation using a fixed very small time step. 


\section{Conclusion}

From this investigation of discretization error, we conclude that both spatial and time discretization error can be minimized by the use of the trapezoidal rule and the surface grid refining algorithms.

\section{References}

1. E.W. Scheckler and A.R. Neureuther, NUPAD Workshop, Seattle, p. 9, 1992.

2. J.J. Helmsen, E.W. Scheckler, A.R. Neureuther and C.H. Sequin, NUPAD Workshop, Seattle, p. 3, 1992.

3. E. Strasser and S. Selberherr, A General Simulation Method for Etching and Deposition Processes, SISDEP, Vol. 5, p. 357, Sept 1993.

4. M. Seifert, F. Richter, and R.G. Spallek, Simulation of Sputter Deposition Process by DUPSIM, SISDEP, Vol. 5, p. 197, Sept 1993.

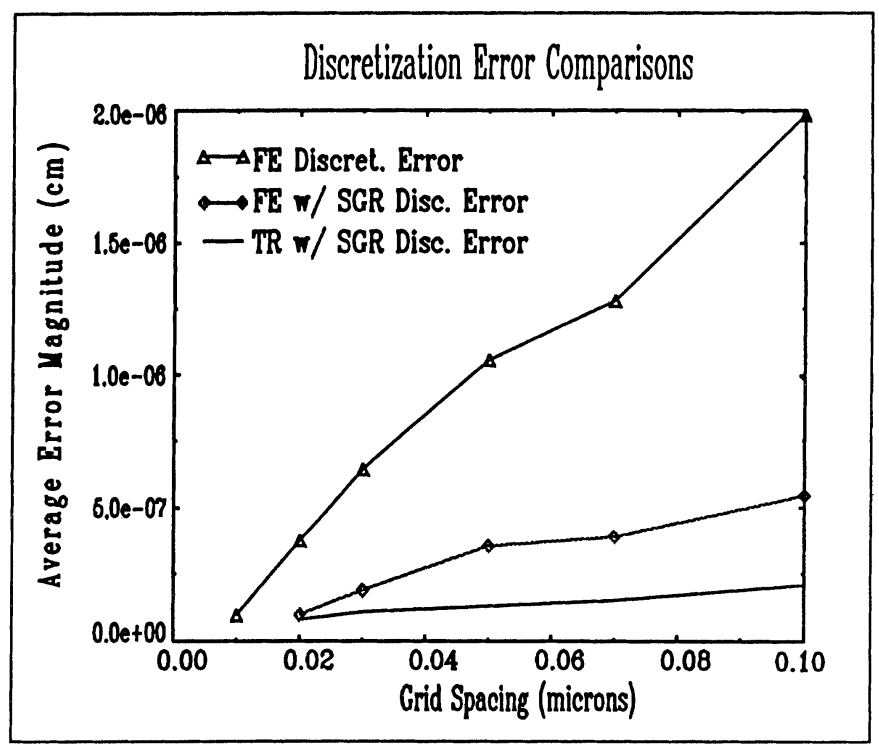

Figure 1. Comparisons of Average Discretization Error vs. Grid Spacing for each algorithm examined. 


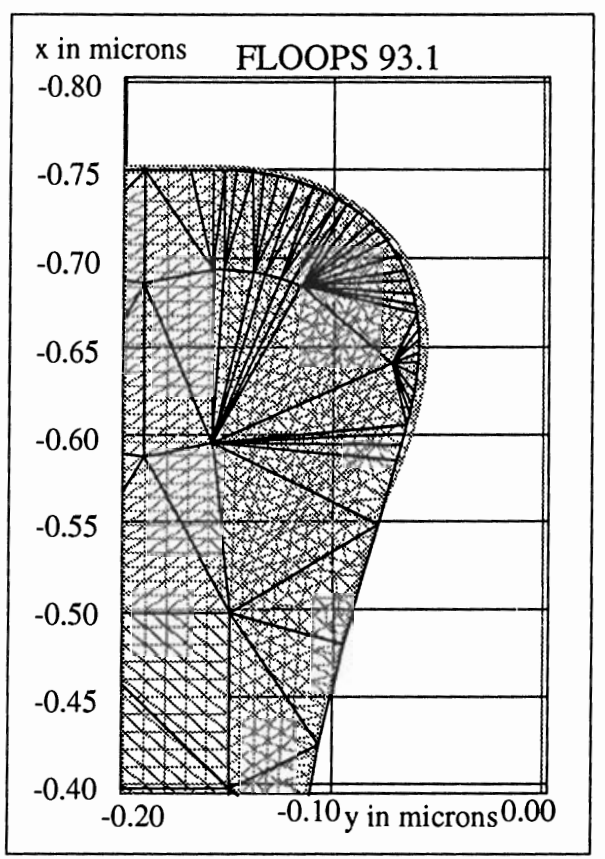

Figure 2.

An anisotropic LPCVD with initial 0.10 um grid spacing simulation with both grid refining and TR time-step control resembles that of an initial 0.01 um grid spacing simulation.

Figure 3.

A surface profile comparison showing how the trapezoidal rule and surface grid refining algorithms reduce the discretization error for coarse grids. $\mathrm{x}$ in microns FLOOPS 93.1

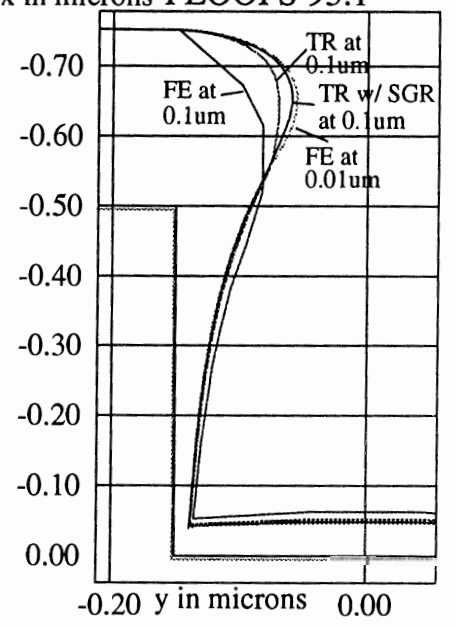

